by Tomas Penaz, Radek Dostal ${ }^{1}$, Isik Yilmaz $^{2}$, Marian Marschalko ${ }^{3}$

\title{
Design and construction of knowledge ontology for thematic cartography domain
}

\author{
${ }^{1}$ VSB-Technical University of Ostrava, Faculty of Mining and Geology, Department of Geoinformatics,17. listopadu 15, 708 33, Ostrava, \\ Czech Republic \\ ${ }^{2}$ Cumhuriyet University, Faculty of Engineering, Department of Geological Engineering, 58140 Sivas, Turkey.E-mail: iyilmaz@cumhuriyet.edu.tr \\ isik.yilmaz@gmail.com \\ ${ }^{3}$ VSB-Technical University of Ostrava, Faculty of Mining and Geology, Institute of Geological Engineering, 17 listopadu 15, 708 33, Ostrava, \\ Czech Republic
}

The paper deals with ontology modelling for the purpose of design and creating of knowledge ontology for thematic cartography. The prepared ontology represents a database of selected declarative cartographical knowledge subsequently employed in the intelligent system for interactive support of thematic map design. The knowledge system pilot project under development is intended for users without necessary cartographical knowledge to whom such system will enable interactive creation of a thematic map and will provide them with support to this aim. The paper brings up information on possibilities of a domain expert (cartographer) in the endeavour to seize domain knowledge of thematic cartography and to express them in a formalized way. OWL ontology concentrates, formalizes and organizes declarative knowledge of thematic cartography domain. The result is the database containing taxonomy of terms hierarchically arranged into categories as well as description of their mutual relations.

\section{Introduction}

The term ontology comes originally from philosophy but this paper does not use the term in its original sense. Here, rather, it relates to "ontology engineering". The relevant technical literature provides a range of definitions of ontology (see, for example, Gruber, 1993; Borst, 1997; Gómez, 1999) which differ considerably from one another. But, for example, one states that an ontogeny is a specification of shared understanding of a knowledge domain that facilitates the accurate and effective communication of meaning (Gruber 1995). This paper refers to ontology engineering and artificial intelligence, and also deals with the term knowledge ontology and related variants.

Knowledge ontologies are the subjects of ontology modelling. They are used as knowledge databases in the process of implementation of intelligent systems in various domains of human activity and make the design of such systems easier (Uschold and
Grüninger, 1996). We need to consider first the cartography domain? Cartography, and thus also thematic cartography, has not been greatly considered by knowledge engineers up to now although developments in the fields of ontologies and artificial intelligence have advanced considerably in work on some cartography solutions. The best known instance of application of ontologies in cartography is probably in cartographic generalization, sometimes referred to as ontology-driven map generalization (Kulik et al., 2005). Ontologies play a significant part in applying processing techniques dealing with contextual generalization of map geographical elements. Firstly, this is a case of line simplification (Kulik et al., 2005) and other aspects of contextual generalization of map features; (Kulik at al., 2005; Edwardes and Dutton, 2006; Lûscher et al., 2007; Regnauld, 2007; Wolf, 2009). An example is the transformation of small scale elements of the map and elimination of less important or unique features applied mostly to spatial data used for visualization in maps published traditional or electronic forms. Thus, related ontologies are aimed at describing taxonomy of meanings, classes and their mutual relations within spatial data.

A further possibility for extending artificial intelligence in cartography is the development of intelligent systems for user-support in Interactive Map Design. One way of using artificial intelligence in this is by optimizing the integration of algorithms into the program code of some program tools. ArcGIS can be used as an example. Jenk's optimizing algorithm (Slocum et al, 2009) enables the user to make an automated design of classes that are classified according to quantitative characters.

Such intelligent systems can also be used as an intermediate in making a cartographic knowledge base suitable for users who lack consistent cartographic education. The intelligent system, by providing a program tool for map design based on previous interaction, can influence the user designing a map in various ways. The simplest is to offer the user elementary recommendations and then to enable the user to enter requirements through a wizard. This paper comes from experience we have obtained during work on a project named Intelligent System for Interactive Support of Thematic Map Design.

Nevertheless, design and setup of an interactively cooperating intelligent system requires the use of special terms in (thematic) cartography. The set of used terms has to be sufficiently developed, accurate and sophisticated so that these cover all relevant issues included in the functionality of the intelligent system. Professional ontology of (thematic) cartography should be appropriate for practical 
implementation of the intelligent system and for the terms of application. However in our literature search we have not found a developed ontology or relevant information on how to approach the design and preparation of ontology of (thematic) cartography. An exception is published signal information (Iosifescu-Enescu and Hurni, 2007) which, however, is not a sufficient starting point for developing this concept. This paper presents some possible approaches to cartographic knowledge formalization, based on ontology engineering, for domain experts. Also, the application of the procedure by which the knowledge database content is transformed in Java interface and classes, is described.

\section{Knowledge of thematic cartography domain}

Knowledge used with regard to of the human cognition-domain is divided traditionally into declarative knowledge and procedural knowledge. The thematic cartography domain is the analogous case.

Declarative (descriptive) knowledge refers to terms used for denomination and description of objects and phenomena in the given domain. This declarative knowledge includes enumeration and definitions of terms, ranking of terms into groups, so-called classes, on the basis of their similarity according to their common features. Further, declarative knowledge deals with the arrangement of terms and classes into hierarchical structures or the expression of differences between various classes according to a specific point of view. Identification of mutual relations amongst pairs of classes, including specific terms, is no less important. Also formulation of relations among synonyms and of cardinal relations among individual classes is included in declarative knowledge.
An example of declarative knowledge in the (thematic) cartography domain is the isolines method which can be defined as a:

“...cartographic method representing certain continuous quantitative characteristics of the phenomenon by lines (isolines) connecting positions with the same intensity. "

This descriptive knowledge includes such features as: contour lines, isobaths, isobars, isotherms, isogons, isohypses, etc. In this connection, the mutual spatial relation of two adjoining contour lines can be described by the statement that adjoining contour lines are not allowed to have any common intersection or point of mutual contact. Another declarative knowledge is set by the use of colour for contour lines. There are many other similar declarative relationships. The final form of an intelligent system application intended to facilitate work with contour lines, including their construction from the point field values and subsequent checking of mutual space relations between them, extends beyond declarative knowledge. It also requires procedural knowledge describing the processing operations.

Problems of formalizing and representing procedural knowledge are beyond the scope of this paper. However a technical solution to the integration of declarative knowledge and procedural knowledge integration is suggested in section 6 (Conclusions and future work).

\section{Representation of thematic cartography declarative knowledge}

The basic problem of incorporating knowledge application into an intelligent program system is to find a way of grasping that knowledge. In the (thematic) cartography domain, it is vital to construct a domain model containing the knowledge base.

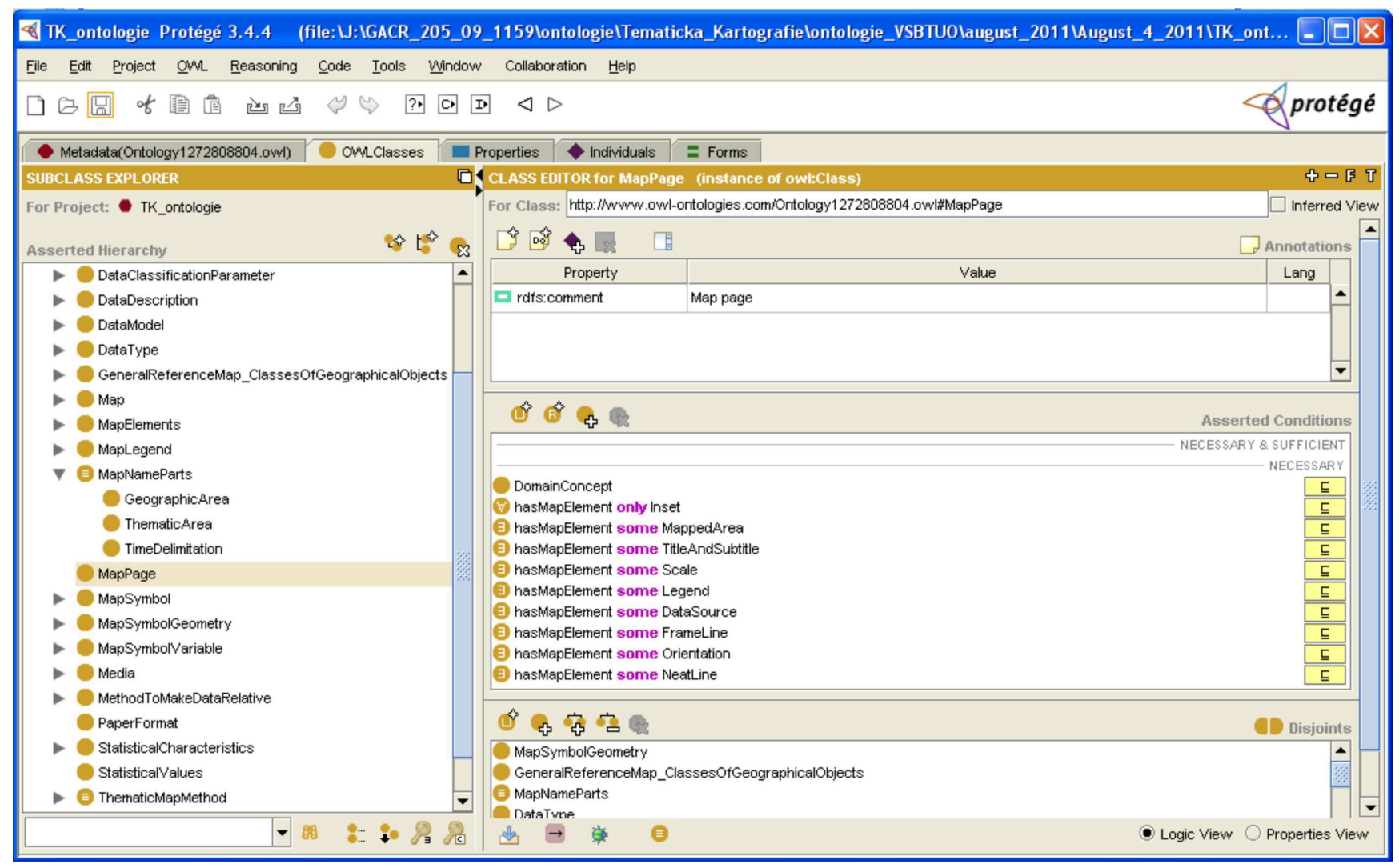

Figure 1. Illustration of Protégé 3.4.4 ontology editor environment. 
In order to be able to work with declarative as well as procedural knowledge in the intelligent program system knowledge needs to be appropriately represented. The term knowledge representation can be seen as satisfying a specific form. It is a case of finding and subsequently using the most appropriate way for knowledge to be formally written. Formalization of expression in (thematic) cartography is a logical continuation of a traditional working method. Both for declarative and procedural domain knowledge there are verified ways of representing information using appropriate languages. When possible, it is advantageous to use some of the standardized representations and tools.

One of the practicable approaches to representation of declarative knowledge is referred to in the literature as knowledge ontologies or just ontologies (Swartout et al, 1996). Ontologies constructed for use in the field of artificial intelligence are hierarchically structured sets of terms (Swartout et al, 1996) which are formal descriptions appropriate for the sharing of knowledge included in them (Gruber, 1993).

Thematic cartography is a discipline in which it is possible to identify a considerable amount of declarative knowledge and therefore subsequently to construct an ontology for part of that domain. But, in available information sources (Iosifescu-Enescu and Hurni, 2007; Kemp, 2004), there are only brief references to domain and professional ontologies covering cartography, thematic cartography and geovisualization.

An important step pursued in ontology engineering (Russell and Norvig, 2009) is domain analysis. Construction of a high quality domain ontology is easier if there is a good theoretical basis for the relevant discipline and if that basis is firmly established in the systems of other disciplines so that relationships can be clarified. Ontology construction can also bring additional value by revealing some inconsistencies theoretical basis of the processed discipline and lead to better formalization of domain knowledge.

In thematic cartography, as in other fields of human activity, disunity of terms and their definitions, of the distributions of terms distributions in hierarchical structures, and coincidences in definitions of mutual relations among the terms can be traced. Disunities often relate to various "cartography schools" established at academic and other specialized institutions in various countries both outside and within the former "Iron Curtain". Before beginning ontology construction, the author must decide which approach towards thematic cartography should be taken.

In the process of construction of any knowledge ontology, it is important to observe the usual sequence of the two basic components known as TBOX (Terminological Components) and $A B O X$ (Assertional Components). The TBOX component comprises the ontology describing the common form of a domain model. It deals with classes, their characteristics and hierarchy (Baader et al, 2007). Only in the next step does it make sense to deal with the ABOX component to with instances of class i.e. specific individual descriptions (Baader et al, 2007). Common recommendations on knowledge ontology construction are available in the literature (Uschold and Grüninger, 1996; Noy and McGuinness 2001).

The main progress in standardization of the tools for ontology creation has been made during the last two decades. The first step was the issue of establishment of a group of languages for the ontologies considered as well as program tools (often called ontologies editors) used by the creator for recording in the relevant language.

On the base of the previous analysis, we decided to record (thematic) cartography ontology in $O W L$ (Web Ontology Language) using the Protégé 3.4.4 editor (Bechhofer, 2004; Horridge et al, 2004) (Fig. 1). One of the reasons for this combination of language and editor application is possibility of the subsequent use of declarative knowledge captured in the ontology. From three feasible species of OWL (known as OWL-Lite, OWL-DL and OWL-Full) (Smith et al, 2004), we chose OWL-DL for the experimental ontology construction. This variant enables the recording of a more comprehensive description of classes defined using expressions written by in description logics-DLs (Baader et al, 2003; Baader et al, 2007; Bechhofer et al, 2004; Russell and Norvig, 2009) which falls within the family of knowledge representation languages (Baader et al, 2007). Also, the OWL "dialect" enables to the researcher to check the consistency of the hierarchy of ontology classes and clears the way for identification of potential deficiencies in the knowledge system of the (thematic) cartography domain.

\section{Construction of ontology}

A simple step in the process of terminological component construction is to formulate term taxonomy (concepts). This is a matter of choosing a set of classes and organizing these into a hierarchical structure ranked from superclass to the subclass and from the more general to the more individual. The aim is to create a structure that can be imagined in the shape of the tree (Fig. 2).

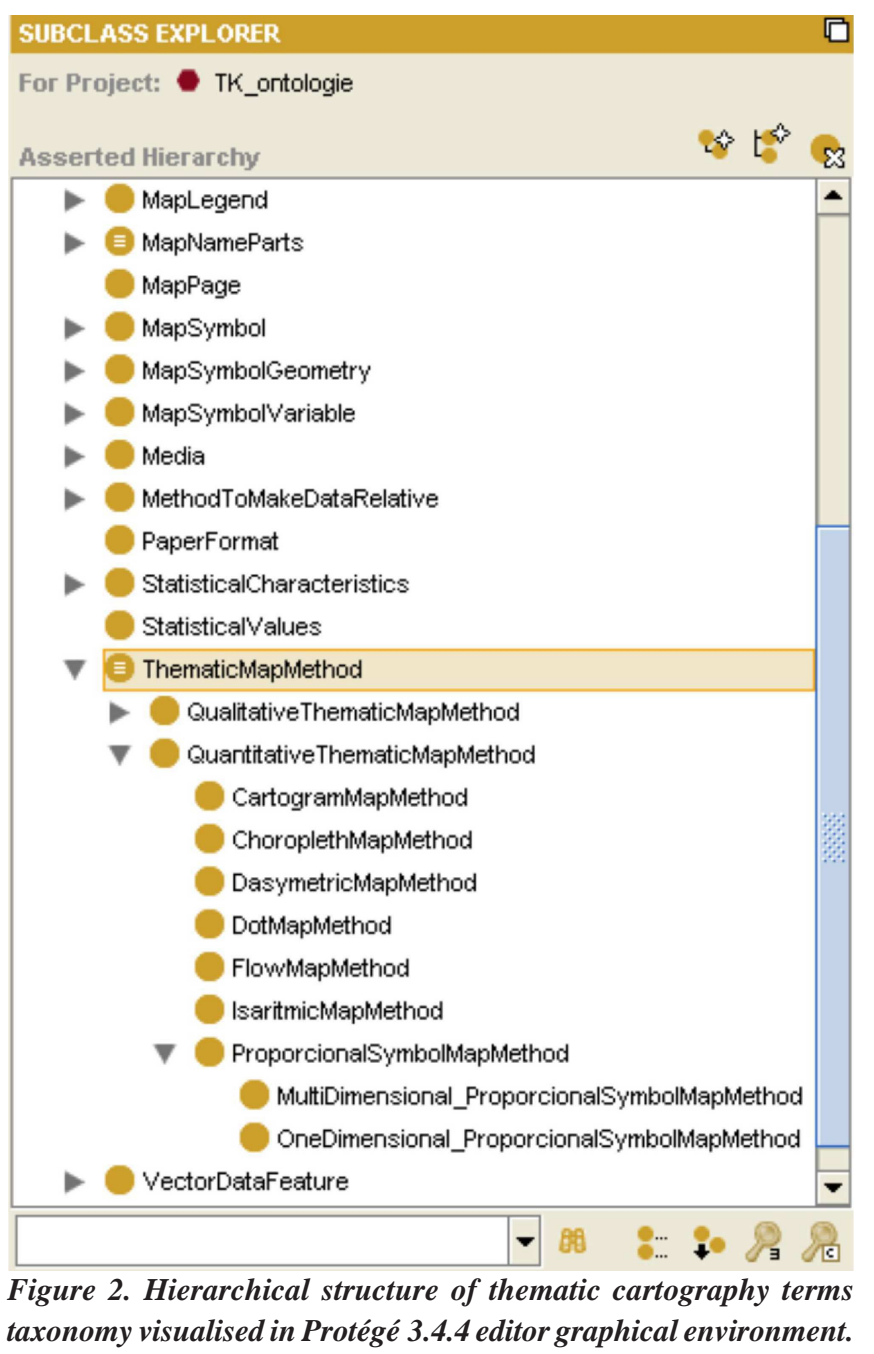


The processor has several types of components available for assembling the basic ontology tree in OWL-DL, the most important of which are class, individual and property.

\section{Class}

Class is a set of individual categories with a common set of properties. In OWL, the class component can be specified by means of six types of class descriptions (Bechhofer 2004):

- a class description (a Uniform Resource Identifier or URI reference);

- an exhaustive enumeration of individuals that together form the instances of a class;

- a property restriction;

- the intersection of two or more class descriptions;

- the union of two or more class descriptions; and

- the complement of a class description.

A named class is characterized as having a URI which identifies it as a unique ontology component (Bechhofer 2004, Russell and Norvig, 2009). When determining URIs of named classes, we recommendation that characters with diacritical marks should not be used. In the created ontology, we applied class names compiled from one or more English words up to several tens of characters in length because of the enhanced information value of longer terms. In the following text, these class names are distinguished by Courier New font. An optional comment attached to individual named classes was used for alternative naming or for description of the meanings of terms in class.

OWL also enables the processor to create anonymous classes which can be imagined as resulting from logical inquiry about named or anonymous classes (Bechhofer 2004). In experimental ontology, we created several anonymous classes formed in connection with some commonly used OWL-DL language constructs. (see Expression of Disjunction)

Constructs for property restrictions are used for specification of conditions by which, on the basis of mutual relation of a chosen pair of classes (object property), the anonymous class of individual factors is assigned (see chapter 4.3 Properties). OWL language constructs define necessary and/or sufficient conditions of the class description. Another type of class descriptor consists of constructs serving as set operators which are it means intersection, union and complement. Through the application of set operators, the use of which is described in the following paragraphs, anonymous complex classes are formed (Smith et al, 2004).

Class descriptors mentioned above are used as the building blocks for defining class axioms which are subsumption, equivalency and disjoint (Baader et al 2007, Bechhofer 2004). The OWL-DL language, in the Protégé environment, allows the mutual relations between named classes to be expressed in terms of the axioms are intended. Another, rather different, type of axiom is the covering axiom which provides relations among classes by means of set operators.

\section{Expression of subsumption}

An example of subsumption axiom in OWL, applied by the rdfs:subClassOf construct, for description of classes relations in

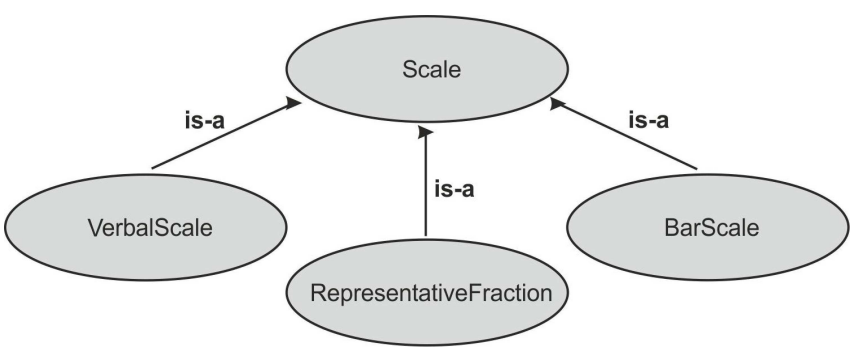

Figure 3. Illustration of subsumption among classes.

thematic cartography domain, is the scale class (scale), within which the following classes are subsumed (Fig. 3) (Slocum et al, 2009):

- RepresentativeFraction (representative fraction);

- VerbalScale (verbal scale); and

- BarScale (bar scale).

The Scale superclass is therefore superior to its RepresentativeFraction, BarScale and VerbalScale subclasses. Relation between two classes can be recorded in two hierarchical levels, where the more general class stands higher in the hierarchy and the more specialised subclass stands lower. The relationship between the more specialised class and the more general class is an IS-A relation ("is the case of") and is characteristic of the BarScale and scale relationship i.e. bar scale is a case of scale. This is a subsumption relationship (Fig. 3).

The formal entry of the subsumption axiom by means Í of DL is as following:

$$
\begin{aligned}
& \text { VerbalScale } \subseteq \text { Scale } \\
& \text { BarScale } \subseteq \text { Scale } \\
& \text { RepresentativeFraction } \subseteq \text { Scale }
\end{aligned}
$$

\section{Expression of equivalence}

The formalized expression of an equivalence axiom, = is going, on the basis owl:equivalentClass construct is:

class description owl:equivalentClass class description

The equivalence axiom can also be connected with the so-called covering axiom of the class. The unification of sibling classes within the superior class is the anonymous class equivalent of the covered class. Thus the equivalence axiom is explicitly described by the relationship of the superior class and the subsumed classes connected by means of a union set operator within the superior class. In the environment of Protégé editor, the user is informed of the constitution of the equivalence relation through a relevant icon (Fig. 5). The explicitly expressed covering class axiom formulated in DL syntax, looks as follows:

$$
\begin{array}{r}
\text { MapNameParts } \equiv \text { ThematicArea } \cup \text { Geographic Area } \cup \\
\text { TimeDelimitation }
\end{array}
$$

A given illustration is described in the section of this paper titled Formulation of relations among classes using set operators. In our experimental ontology of thematic cartography, we have used the equivalence axiom application several times within the context of covering classes. 


\section{Expression of disjoint}

The application of the disjoint axiom using the owl:disjointWith construct can be expressed as:

\section{class description owl:disjointWith class description}

The disjoint axiom is needed for use for each subclass of the given class. In fact, the subclass disjoint in OWL is not expressed implicitly and union of subclasses in OWL does not cover the superclass (Smith et al, 2004). Therefore the author of the ontology has to determine the disjoint of subclasses explicitly and, in this way, eliminate classes that could be partially or completely identical to another sibling class or classes. So the expression of disjoint is an important axiom describing class relations and, sometimes, relationships to other sibling classes. By using the class disjoint it is possible to express that an individual or an object cannot be the instance of more than one of those classes. This way, it is possible to define semantically different terms within the class.

The following building block from experimental ontology applies the disjoint axiom among subsumed classes of Quantitative ThematicMapMethod class. The construction expresses the disjoint of DotMapMethod class toward all sibling classes.

\section{<owl:Class rdf:about="\#DotMapMethod"> \\ $<$ rdfs:subClassOf rdf:resource="\#QuantitativeThematicMap Method"/> \\ <rdfs:comment rdf:datatype="http://www.w3.org/2001/XML \\ Schema\#string" $>$ Metoda tecek $</$ rdfs:comment $>$}

$<$ owl:disjointWith rdf:resource="\#FlowMapMethod"/>

<owl:disjointWith rdf:resource="\#ChoroplethMapMethod"/>

<owl:disjointWith rdf:resource="\#DasymetricMapMethod"/> <owl:disjointWith rdf:resource="\#IsaritmicMapMethod"/> <owl:disjointWith rdf:resource="\#ProporcionalSymbolMap

Method"/>

<owl:disjointWith rdf:resource="\#MultivariateMapMethod"/> <owl:disjointWith rdf:resource="\#CartogramMethod"/> $</$ owl:Class>

In experimental ontology, we also specified disjoint to express relationships among TitleAndSubtitle, MappedArea, Legend, Scale, Inset, DataSource, Orientation, and FrameLineAndNeatline classes
(Slocum et al, 2009; Peterson, 2009). In this way, semantic differences between individual composition map elements is formalized. It is the case that a particular individual within the class of composition map elements cannot both represent the legend and, at the same time, the scale of the map (Fig. 4).

The following example of a record in DL syntax illustrates the application of the disjoint axiom and the way it eliminates the parallel pertinence of an individual element to the Legend and Scale subclasses. The $\neg$ symbol expresses complement or, rather, logical negation, while the Í symbol expresses subsumption.

A special type of class description connected with relations of individuals to individuals of another class, are so-called property restrictions and embraces the anonymous classes of all individuals meeting the restrictions (Bechhofer, 2004). Problems of property restrictions are explained in section 4.3 of this paper.

\section{Expression of enumeration}

Another class descriptor is enumeration (expressed by owl:oneOf construct) of individuals (see section 4.2) which are instances of class (Bechhofer, 2004). An example of enumeration we used in experimental ontology is the enumeration of normalized paper size in the series $\mathrm{A}(\mathrm{A} 0, \mathrm{~A} 1, \mathrm{~A} 2, \mathrm{~A} 3, \mathrm{~A} 4, \mathrm{~A} 5)$ :

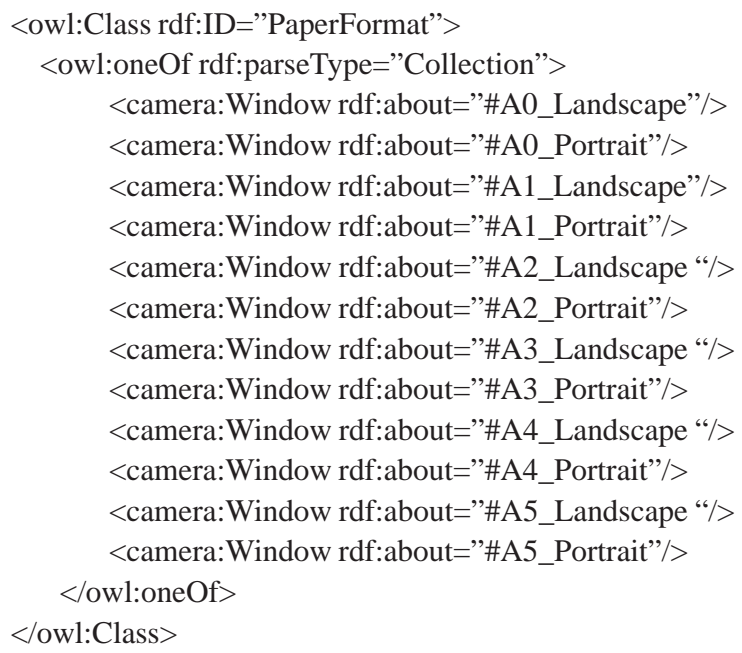

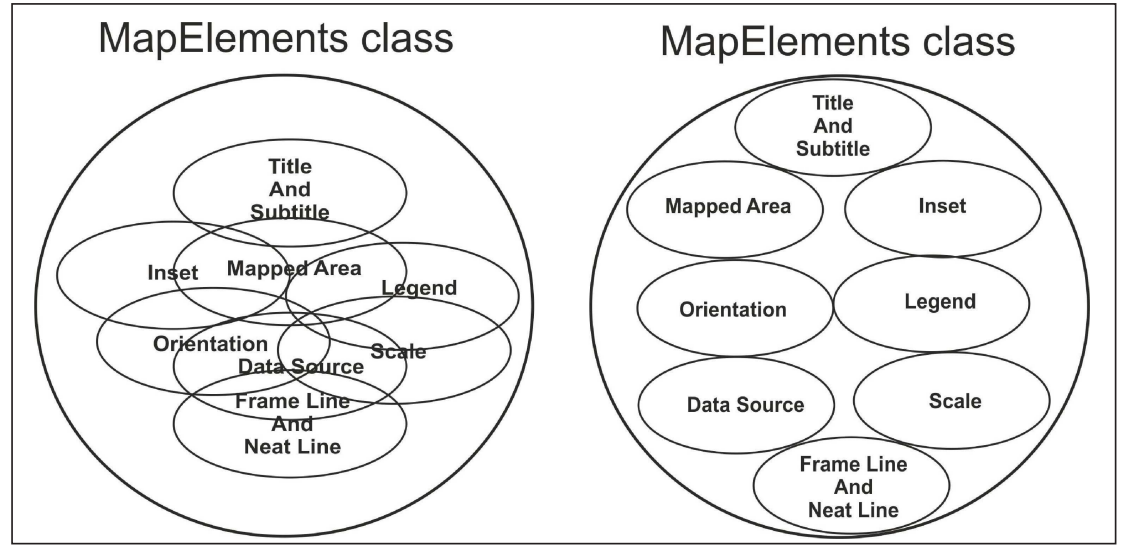

Figure 4. Illustration of disjoint axiom function for distinguishing of class meaning map composition elements. OWLclasses TitleAndSubtitle, MappedArea, Legend, Scale, Inset, DataSource, Orientation, FrameLineAndNeatLine on the left are assumed to "overlap". In order to "separate" the same group of classes on the right we made them disjointed from one another.

\section{Expression of relations among classes using set operators}

Set operators, including intersection, union and complement, are intended to connect named or anonymous classes by means of logical queries resulting in another anonymous class.

Set operator intersection is applied by the owl:intersectionOf construct. At the time of preparing this paper we had not found any meaningful use for this operator in experimental ontology of thematic cartography.

Set operator union, in OWL expressed by owl:unionOf construct, is often used for covering axiom expression. The first part of the covering axiom is the class which is covered, and the second part consists of the classes which form the covering (Horridge et al, 2004). We used the covering axiom 


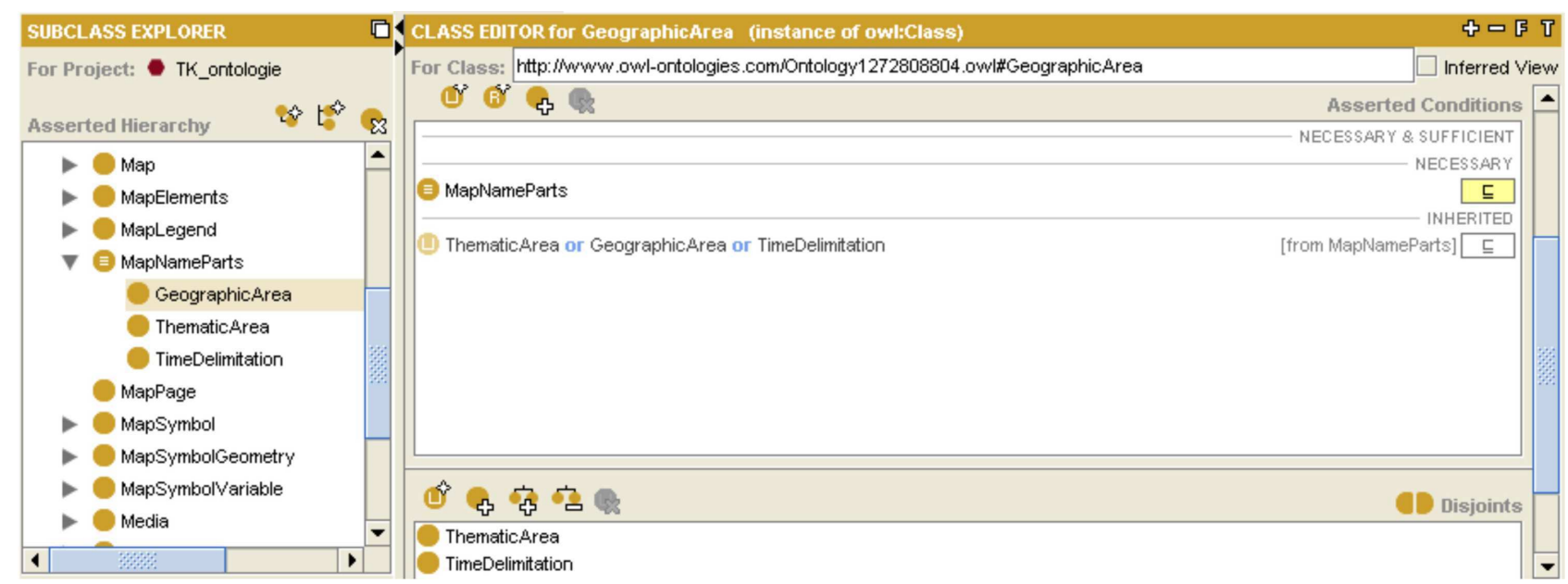

Figure 5. Protégé 4.4 .3 editor environment with illustration of the definition of MapNameParts class covering axiom

in our experimental ontology of (thematic) cartography to formalize the entry of cartography knowledge that the map name may contain thematic area, geographic area and time delimitation (Fig. 5). The MapNameParts class is super-ordinated to three subsumed classes which completely cover the superclass (Fig 6). One component of the covering axiom formalized entry in DL syntax is the symbol $=$ for expression of equivalence and the symbol $\cup$ for union.

\section{MapNameParts class}
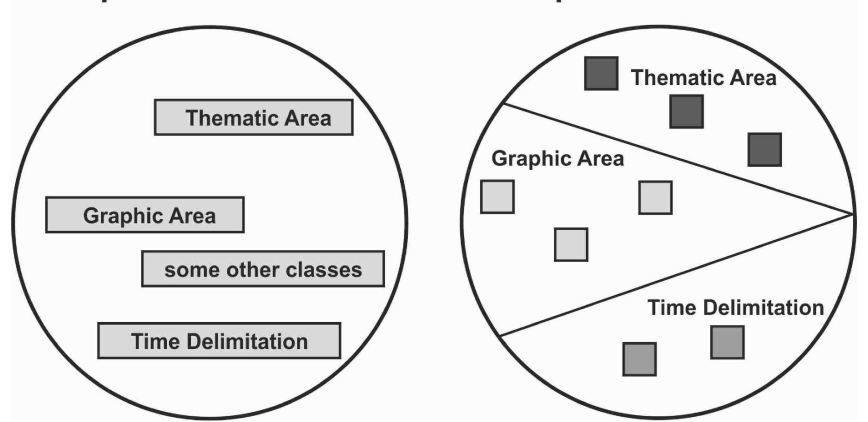

Figure 6. The effect of using a covering axiom on the MapName Parts class. OWL classes ThematicArea, GeographicArea, TimeDelimitation on the left without a covering axiom. With a covering axiom on the right, MapNameParts class is covered by ThematicArea, GeographicArea, TimeDelimitation classes.

It is impossible that, except for the ThematicArea, GeographicArea and TimeDelimination classes, any other class could be subsumed to MapNameParts class.

$$
\begin{array}{r}
\text { MapName } \equiv \text { ThematicArea } \cup \text { GeographicArea } \cap \\
\text { TimeDelimitation }
\end{array}
$$

A covering axiom can also be used to express the mutual relation of QuantitativeThematicMapMethod and QualitativeThematicMap Method classes or the relation of these classes to the superior ThematicMapMethod class.

$$
\begin{array}{r}
\text { ThematicMapMethod } \equiv \text { QuantitativeThematicMapMethod } \cup \\
\text { QuantitativeThematicMapMethod }
\end{array}
$$

A covering axiom can also express relation of subsumed classes, such as the Red, Green, Blue colour compounds, to the superior Colour class and thus explicitly eliminate possibility that any other subclass (colour compound) may be involved in colour creation. At the same time, a union operator expresses acquaintance with the resulting colour mixing from three colour compounds:

$$
\text { Color } \equiv \text { Red } \cup \text { Green } \cup \text { Blue }
$$

A set operator complement applied by means of owl:complement Of construct describes a class by expression of all individuals that do not fall into the described class. This represents an analogy to logical negation in the case of Boolean expression of the classes. In the experimental ontology, we used a set operation implement for cartogram method elimination in the creation of a choropleth map. The following OWL code illustration requires knowledge of problems associated with object properties (see section on Properties):

<owl:Class rdf:about="\#ChoroplethMap"> <rdfs:comment rdf:datatype="http://www.w3.org/2001/

XMLSchema\#string" $>$ Kartogram $</$ rdfs:comment $>$ $<$ owl:disjointWith rdf:resource="\#ProporcionalSymbolMap"/> <owl:disjointWith rdf:resource="\#Cartogram"/> $<$ rdfs:subClassOf rdf:resource="\#QuantitativeThematicMap"/> $\langle$ rdfs:subClassOf>

$<$ owl:Restriction>

<owl:onProperty>

<owl:ObjectProperty rdf:ID="usesQuantitativeMap Method"/>

$</$ owl:onProperty>

<owl:someValuesFrom rdf:resource="\#ChoroplethMap Method"/>

$</$ owl:Restriction $>$

$</$ rdfs:subClassOf $>$

$\langle$ rdfs:subClassOf $>$

<owl:Class>

<owl:complementOf>

<owl:Restriction>

$<$ owl:onProperty>

<owl:ObjectProperty rdf:ID="usesMapMethod"/>

$</$ owl:onProperty $>$

<owl:someValuesFrom>

<owl:Class rdf:about="\#CartogramMapMethod"/>

$</$ owl:someValuesFrom $>$

$</$ owl:Restriction $>$

$</$ owl:complementOf $>$

$</$ owl:Class $>$

$</$ rdfs:subClassOf $>$

$</$ owl:Class $>$ 


\section{Individual}

The component individual is used to represent a particular domain object which falls into a specific class. The component class and, likewise, the component individual is identified with its name with regard also to the sense of the identifier of the individual (URI). The URI identifies the individual as the unique ontology component. An example of an individual is Thames (the River Thames) falling into the Stream class (watercourse).

In some cases, it is difficult to determine whether a class or an individual is meant. An example can be seen in NorthArrow (direction sign) which, in the first case, can be described as an individual falling into MapElements class. Under certain circumstances, however, it is correct to speak of NorthArrow as a class covering a wide range of individuals which are particular styles for plotting the direction sign on a map. This second interpretation of the NorthArrow object makes sense when the user has chosen particular style offered by a tool for interactive map design. Boundaries between the terms class individual are made according to the particular application of constructed ontology (Noy and McGuinness, 2001). The individual is incorporated into ontology components primarily in those cases when it is necessary to define the particular class by means of that individual (Svátek and Labský, 2003).

In the experimental ontology of (thematic) cartography within the project, we defined, for PaperFormat class, components of the type of individual to include dimensions of standardized formats of traditional carriers - sheets of paper - into the declarative knowledge file. The PaperFormat class is described with datatype (see section 4.3) properties dimension_height and dimension_width, for numerical values of standardized formats. The defined A4_Landscape, A4_Portrait, A3_Landscape, A3_Portrait individuals carry values of given formats in terms of dimension_height and dimension_width datatype properties:

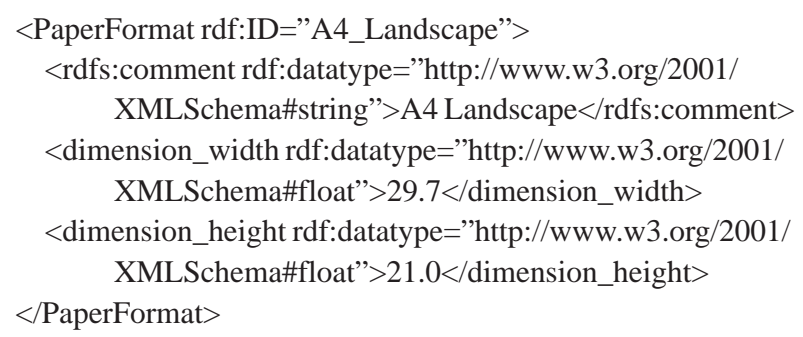

\section{Properties}

Properties are important compounds of ontology because they express binary relations between two individuals (Smith et al, 2004). OWL-DL facilitates work with three types of properties. Two principal types of properties are object properties and datatype properties. Another type is comment properties which allows a "string" of metadata to be assigned to classes, individuals, and object and datatype properties (Horridge et al, 2004).

The component class and object, datatype and comment properties are each determined by names which are unique identifier URIs. For property definition, a property ID is required (Bechhofer 2004) which, again, is a URI. This way, a property becomes a unique component of ontology. In URI determination of object, datatype and comment properties, characters with diacritical marks were not used. For properties definition, strings up to several tens of characters long were used for naming to give these identifiers informational value that could be readily understandable by a cartographer with knowledge of technical English.

\section{Object property}

An Object property represents the relation among individuals of two classes (Smith et al, 2004). To ensure that a designed object property is sensible, two existing classes, known as $D(f)$ property domain and $H(f)$ property range are used to establish the binary relationship between any two individuals. Relation formalization can be verbally expressed as, for example, "it is comprised of"or "it consists of". An example is the hasMapElement object property which creates binary relationships between individuals of the MapPage class, ThematicMapPage and GeneralReferenceMapPage, and individuals of MapElements class which include TitleAndSubtitle, MappedArea, Legend, Scale, Inset, DataSource, Orientation, FrameLineAndNeatLine.

So, through the hasMapElement object property, the formal expression of the set of knowledge contained in one of the basic rules of (thematic) cartography requiring that the (thematic) map page may contain composition elements (title and subtitle, mapped area, legend, scale, inset, data source, orientation, frame line and neat line) can be achieved (Slocum et al, 2009; Peterson, 2009) (Fig. 7). The following presentation of OWL-DL code expresses how, by means of the hasMapElement object property, the binary relation among individuals of MapPage class and Legend individual from MapElements superclass is implemented:

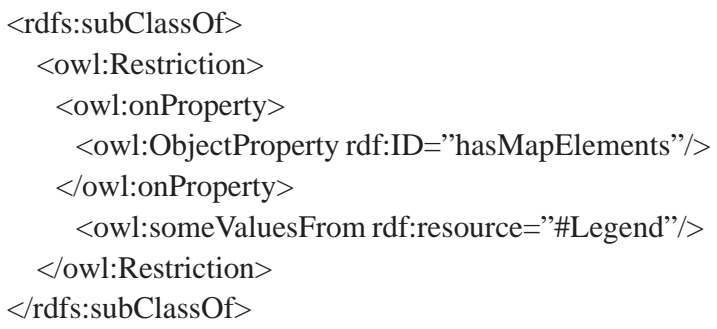

A corresponding inverse property can be defined for every object property (Smith et al, 2004; Horridge et al, 2004). If some of the object properties set a relation between individuals A and B, the inverse property creates a relation between $\mathrm{B}$ and $\mathrm{A}$. The relation formalization can be verbally expressed as, for instance, "it is a compound of". An example is the inverse object property isMapElementOf (Fig. 7).

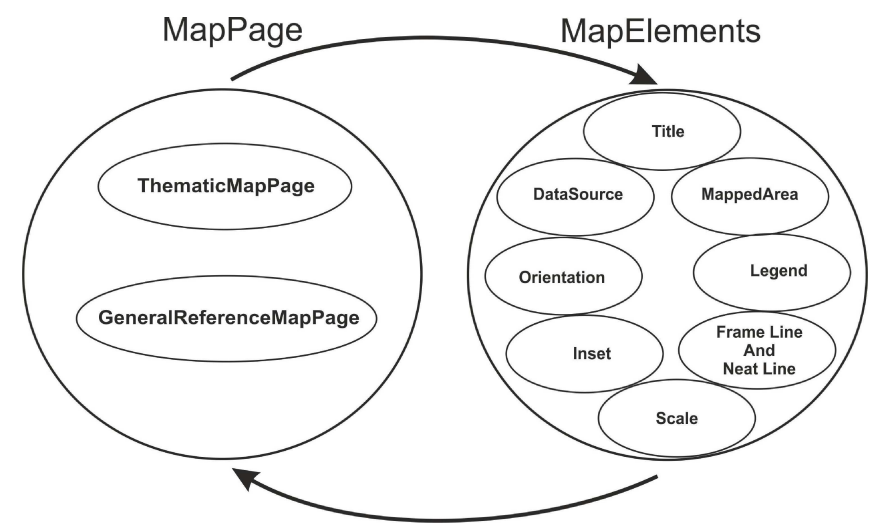

Figure 7. Schematic illustration of hasMapElement object property expressing existence of basic composition elements on a map page using isMapElementOf inverse object property. 
Through this inverse object property, a set of knowledge specifying the meaning of individual composition elements (Title, MappedArea, Legend, Scale, Inset, Data Source, Orientation, FrameLineandNeat Line) for individuals of MapPage class is formally expressed. In other words, the inverse object property isMapElementOf expresses compositional elements that occur on the map page.

Object properties can be hierarchically structured as can classes. Also for object properties, the relation of sumption between object superproperty and subsumed object property is valid. This way, and object properties taxonomy is created where the more general object property is superior to the more specialised subsumed object subproperty. An example is the hasParts object property which is superior to the more specific hasMapElement and hasMapNameParts object properties.

The OWL language component of the sense of properties component can further be specified by means of $O W L$ property characteristics (Smith, 2004 et al; Horridge et al, 2004). In this connection, object properties can be further defined as:

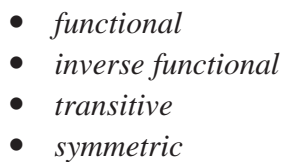

If for the given individual element, by means of object property, a maximum relation to another individual can be created, this object property can be defined as a functional (or single valued) one. An example of a functional object property is hasLegend which expresses the relationship between the MappedArea and the Legend and reflects the fact that the particular mapped area can have only one legend (although that can be divided into several individual parts).

In the ontology, an inverse functional object property can be created for each object property. Thus, the defined object property represents a parallel to the functional object property. So, for instance, for the hasLegend object property it is possible to create the inverse functional object property isLegendOf. Through this functional object property there is formally presented knowledge expressing relation of Legend individual which can be a specific legend, to MappedArea individual which is a map field of a specific thematic map. So, the particular legend has a relation to the particular map field. Thus, functional object property and inverse object property have a reciprocal relation defined by cardinality $1: 1$.

Another potential feature of object property is transitivity. For a transitive object property which creates a relationship between individuals A and B individuals and a further relation between B and $\mathrm{C}$ individuals, it is possible to establish that the same relation also occurs between individuals A and C (Smith et al, 2004; Horridge et al, 2004). For example, the isSubregionOf object property between regions is transitive (Bechhofer, 2004):

\section{<owl:TransitiveProperty rdf:ID="isSubregionOf"> $<$ rdfs:domain rdf:resource="\#Region"/> $<$ rdfs:range rdf:resource="\#Region"/> \\ $</$ owl:TransitiveProperty>}

If, for instance, the transitive object property, SubregionOf, expresses the relation between individuals ArizonaState and UnitedStates and between the individuals MaricopaCounty and ArizonaState from the Region class, the OWL reasoner should be able to derive that if MaricopaCounty, ArizonaState and UnitedStates are regions, and MaricopaCounty is a subregion of ArizonaState, and ArizonaState is a subregion of UnitedStates, then MaricopaCounty is also a subregion of UnitedStates.

Thus, this ontology component complex expresses declarative cartography knowledge which can be put as following:

"Maricopa County is located in the State of Arizona and the State of Arizona is located in the United States."

On the basis of these transitive object properties, the declarative cartography knowledge is derived:

\section{"Maricopa County is located in the United States."}

The last feature of object property is symmetry. If object property $\mathrm{P}$ is defined as the symmetric object property and this object property expresses the relation between individuals $\mathrm{A}$ and $\mathrm{B}$, then the same object property expresses a similar relation between individuals $\mathrm{B}$ and A (Smith et al, 2004; Horridge et al, 2004). An example is the symmetric object property neighbourOf which expresses the relationship between individual map sheet $\mathrm{A}$ and adjacent map sheet B. The identical symmetrical object property neighbourOf then also expresses the symmetrical relationship between the map sheet B and adjacent map sheet $\mathrm{A}$. In other words, if the map sheet $\mathrm{A}$ is adjacent to the map sheet $\mathrm{B}$, then also the map sheet $\mathrm{B}$ is adjacent to the map sheet A. The neighbourOf symmetric object property was prepared which describes the relation between individuals of the MapSheets class:

$$
\begin{aligned}
& \text { <owl:SymmetricProperty rdf:ID="neighbourOf"> } \\
& \text { <rdfs:domain rdf:resource="\#MapSheets"/> } \\
& \text { <rdfs:range rdf:resource="\#MapSheets"/> } \\
& \text { </owl:SymmetricProperty> }
\end{aligned}
$$

\section{Property restrictions}

If mutual relations among individuals are to be described with precision in the ontology, it is necessary to use a property restrictions mechanism (Bechhofer, 2004; Smith et al, 2004). The constitution of an object property and the subsequent determination of a property domain and range for this property loosely set relations between two individuals. Sometimes global property restriction is stated (Lacy, 2005; Svátek et al, 2009). On the basis of global restriction of the isMapElementof property, it is evident that a relationship among individuals of MapPage class and Legend, Scale, Title and other individuals from MapElements superclass does exist.

On the basis of a properties restriction mechanism, the conditions through which the property in question creates a relation among individuals can be described even more completely. Using appropriate conditions, it is possible, for example, to arrange a cartography rule requiring that the map may compulsorily contain certain usual compositional elements, namely mapped area, title, legend, scale and imprint. The aim of the MapPage class description is to define the case of a primitive class for which necessary conditions are specified (Fig. 8), or, better, to define a class for which necessary and sufficient conditions are specified (Smith et al, 2004). At present, in our experimental ontology of (thematic) cartography, one defined class exists, specifically the ThematicMap class, but the conditions for it are not yet sufficiently determined and formulated.

If the object property is to depict class semantics, it is necessary to use some local property restrictions (Lacy, 2005) which, in OWL are divided into two basic types (Horridge et al, 2004). 
- value constraint

- cardinality constraint

Value constraint can be further more precisely specified by means of:

- value constraint owl:allValuesFrom

- value constraint owl:someValuesFrom

- value constraint owl:hasValue

Value restriction, a so-called existential quantifier, in introduced in formal OWL by the owl:someValuesFrom construct which, in formal DL, is expressed by the symbol $\$$. This can be expressed using a sentence beginning with words such as some, at least 1, or some values from. Then, using an existential restriction, it is possible to express, in a formalized way, declarative knowledge (Slocum et al., 2009) which in natural language says that "map page ..."

“...contains at least one element of map composition which is the mapped area."

In OWL-DL syntax, the same cartographic rule says:

$$
\exists \text { hasMapElement some Mapped Area }
$$

However, one basic cartographic rule, it that this is not sufficient for absolute enumeration of basic elements which usually can be found on a map sheet. Therefore, other similar basic rules should be formulated:

„... it contains at least one element of map composition which is scale,"

„... it contains at least one element of map composition which is title and subtitle,"

„... it contains at least one element of map composition which is frame line and/or neat line, "

„,...it contains at least one element of map composition which is data source, "

„... it contains at least one element of map composition which is orientation, "

„... it contains at least one element of map composition which is legend."

Since this formulation of cartographic rules was undertaken in a Protégé 3.4.4 environment connected by AND logical operators, the compound rule describing the map page could be created (Fig. 8).

Value restrictions, also called universal restrictions, in OWL are introduced by the owl:allValuesFrom construct and, in formal DL, is introduced by the symbol ". In plain language these usually begin with words such as only, no values except, only values from. The universal restriction of the object property allows only an optional relation between two classes, not an obligatory one.

An example of using of this axiom is restriction of the object property hasMapKey, that combines the class TopographicMap only with the class TopographicMapSymbols. It is possible to write the same knowledge in OWL-DL syntax, combining of universal restriction and existential restriction, as follows:

hasMapKey only TopographicMapSymbols

hasMapKey some TopographicMapSymbols

The object property hasMapKey without axiom closures allows the establishment of relationships of the individual class TopographicMap to individuals which are not instances of the class TopographicMapSymbols. Thus the topographic map could relate this to geological symbols (individuals of class GeologicalMapSymbols) but that is not desirable because they are designed for geological maps.

Cardinality restriction of the object property enables more accurate specification of the number of relationships between the particular class individual and a second property which is a part of the object property definition. The following types of cardinality restrictions are available:

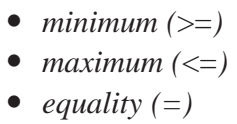

In this experimental ontology, we used cardinality restrictions for specification of a number of classes at classification in terms of quantitative characteristics. The statistical file made for choropleth map design can be used to make a minimal classification into two classes but a maximum of 6 classes. Cardinality restriction of a minimal number of classes eliminated the possibility of classification into one class because it made no sense. The maximum number of classes was restricted to 6 because higher number of classes, subsequently represented by a quantitative colour scale, could handicap persons with colour vision deficiency.

We have not used the value restriction using owl:has Value construct restriction in the experimental ontology of thematic cartography so far.

\section{Datatype property}

Datatype property is relation between an individual and a datatype defined according XML schema datatype value (Smith et al, 2004; Biron and Malhotra, 2004) or relation between an individual and

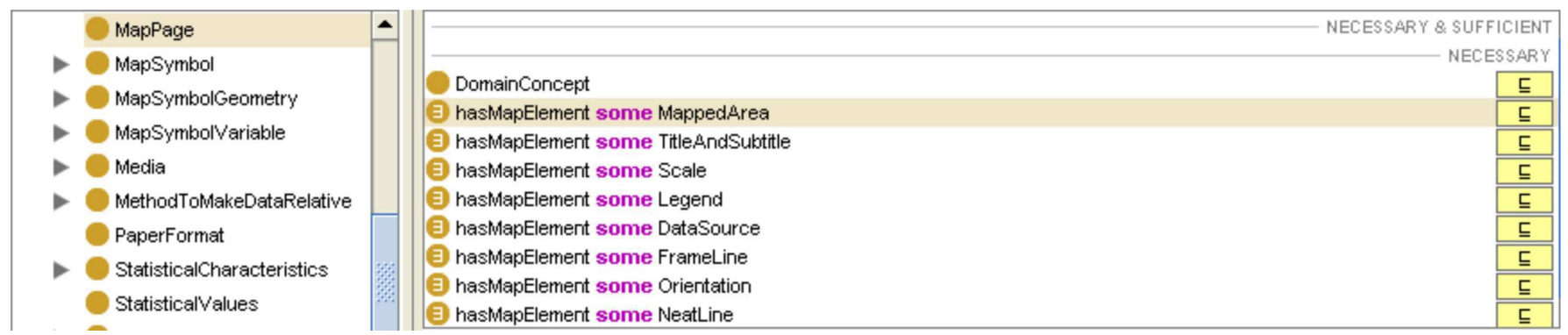

Figure 8. Protégé environment and formalized expression of obligatory assignment of the basic elements on a map sheet using existential property restriction. 


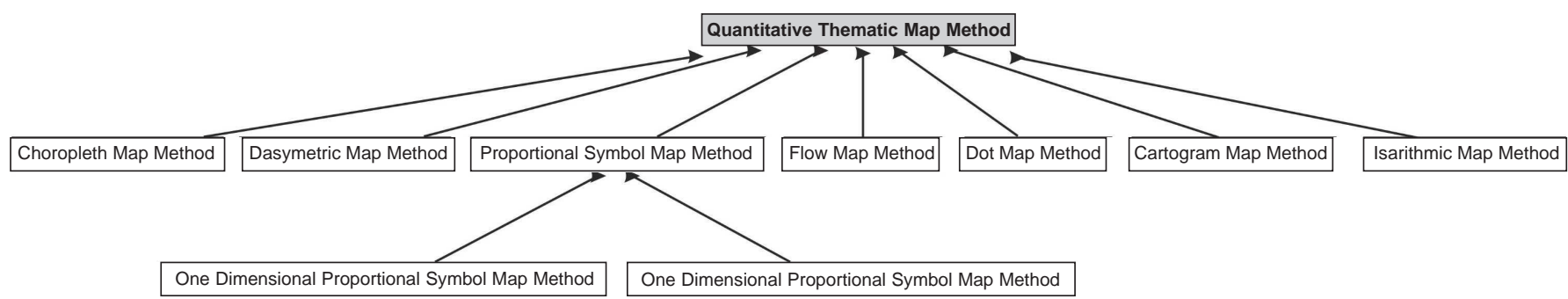

Figure 9. Visualization of classes hierarchy segment in experimental ontology executed using OWLWIZ module.

Resource Description Framework (RDF) literal (Smith et al, 2004).

In practice, datatype property is useful for assigning the value field to any individual within defined classes. In this experimental ontology, we further used datatype properties for formalization of knowledge expressing dimensions of pages of normalized formats (A0, A1, A2, A3, A4, A5). For PaperFormat class we defined data type properties dimension_height and dimension_width of a single float type. For each of the formats we defined two individuals in OWL, for example A4_Portrait and A4_Landscape, and assigned real number values of both dimensions in centimetres to each of them (see section 4.2). For example:

<PaperFormat rdf:ID="A4_Landscape">

$<$ dimension_height rdf:datatype="http://www.w3.org/2001/

XMLSchema\#float">21.0</dimension_height $>$

$<$ dimension_width rdf:datatype="http://www.w3.org/2001/

XMLSchema\#float">29.7</dimension_width>

$</$ PaperFormat $>$

\section{Ontology visualization}

One of the advantages brought by available ontology engineering tools is the possibility of visualizing potential outcomes of ontology construction. These options are also offered by Protégé 3.4.4, the graphic user environment of which enables visualization of the hierarchical structure of the components used; classes, object properties and individuals. Another highly illustrative way of visualization (Fig. 9) is feasible using OWLWIZ plug-in.

\section{Conclusions and recommendations}

On the basis of our previous work on the construction of experimental domain ontology of (thematic) cartography, we have been able to verify possibilities of declarative knowledge representation using OWL. We also carried out the transformation of experimental ontology on the base of declarative knowledge recorded in the form of interfaces and classes entered in Java. The purpose of the transformation was to obtain a source code containing a description of the previously created declarative knowledge base. The partial aim of the experiments was to check potential loss of information which might occur as a consequence of transformation.

We have discovered that a range of declarative knowledge can be expressed using OWL components. During the process of experimental ontology construction, we used a set of chosen terms from the (thematic) cartography domain connected with map creation using a choropleth map and a diagram map. The resulting experimental ontology contains class definitions and their hierarchy. Through object properties, we expressed relationships between the defined classes of cartographic domain because the constructs of the OWL-DL dialect enable semantics of the defined classes to be depicted more accurately. The ontology creator should use as many required property restrictions as possible (global restriction, local restriction, existential restriction, cardinality restriction and universal restriction). Identified datatype properties enable the creator to specify classes and to determine the data type more completely and accurately.

During ontology construction, we took account of some generally valid and important recommendations (Noy and McGuinness, 2001) with regard to identifier determination. When choosing a URI identifier string of all types of ontology components, we also avoided using a numeral in the position of the first character because, after ontology components are transformed into interfaces and Java classes, classes and interfaces beginning with a numeral would have been created which do not conform to Java syntactic rules and could not be used.

Knowledge ontology construction is an exacting activity, the success of which depends on many factors. The objective factor is represented by the situation in which the given discipline has been established within the system of other disciplines. Related to this is the quality of the conceptual model by which the given discipline is described. Problems related to transformation of the conceptual model into the form of logical model recorded using OWL-DL, are often connected with imperfections of the existing conceptual model which frequently lie within the scope of the discipline. The causes of problems connected with ontology construction are is imperfection, obsolescence or even absence of valid standards. Standards connected with terminology and term hierarchy play an important role.

In the process of ontology, the form is also influenced by subjective factors dependent on the personality of the ontology creator.

The aim of the further experiments will be to verify the program application so that the declarative knowledge base and the procedural knowledge base can be integrated. Through transformation of the created experimental domain model into Java language, we will subsequently establish whether the resulting description of the declarative knowledge base from the thematic cartography domain can be integrated with the procedural knowledge stored in the file of cartographic rules. In this connection, we chose the Java language because it is connected with environment of the DROOLS software system which will be used for further development of the intelligent system within the project.

We chose DROOLS system, which is a Business Rule Management System (BRMS), on the basis of the preceding comparative study. For this study, the Expert System Shell is needed because it is suitable for development of the intelligent system for thematic maps with interactive support. The DROOLS tool is the plug-in of the Eclipse programming environment in which the further development of the intelligent system is under way. We plan to check the export of the logical domain stored in OWL-DL into the Java 
code and alternatively by means of RDFReactor, OwlJava and Protégé OWL-API plug-ins. At the same time, we will run other experiments on ontology transformation into Java program code with the aim to minimize information loss. For this purpose, we will try to use some alternative conversion tools the use of which has been referred to favourably in recent publications (Stevenson, 2011). More detailed information can be found on the web pages of our project (http:// cartoexpert.comuf.com/).

\section{Acknowledgement}

This material is a part of "Intelligent System for Interactive Support of Thematic Map Design" project supported by the Czech Science Foundation under Grant No. 205/09/1159. Support of the Czech Science Foundation is gratefully acknowledged.

\section{References}

Baader, F., Calvanese, D., McGuiness, D., Nardi, D., Patel-Schneider, P. (eds), 2003. The Description Logic Handbook: Theory, Implementation and Applications. Cambridge University Press, Cambridge.

Baader, F., Horrocks, I., Sattler, U., 2007. Chapter 3 Description Logics. In: van Harmelen F., Lifschitz V., Porter B. (eds.), Handbook of Knowledge Representation. Elsevier Science San Diego.http://www.comlab.ox.ac.uk/ people/ian.horrocks/Publications/download/2007/BaHS07a.pdf

Bechhofer, S., 2004. OWL Web Ontology Language. Reference. W3C Recommendation 10 February. http://www.w3.org/TR/owl-ref/

Biron, P.V., Malhotra, A., 2004. XML Schema Part 2: Datatypes Second Edition. W3C Recommendation. http://www.w3.org/TR/2004/RECxmlschema-2-20041028/

Borst, W.N., 1997. Construction of Engineering Ontologies for Knowledge Sharing and Reuse. PhD dissertation, University of Twente, Enschede, CTIT Ph. D-series No. 97-14, http://doc.utwente.nl/17864/1/ t0000004.pdf

Edwardes, A., Dutton, G., 2006. Ontological Modelling of Geographical Relationships for Map Generalization. In: Proc. of Workshop of the ICA Commission on Map Generalization and Multiple Representation - June 25th.

Gómez, P.A., Benjamins, V.R., 1999. Overview of Knowledge Sharing and Reuse Components: Ontologies and Problem-Solving Methods. In: Proc. of IJCAI-99 Workshop on Ontologies and Problem-Solving Methods: Lessons Learned and Future Trends, CEUR Publications, Vol. 18, and University of Amsterdam, Amsterdam. http://sunsite.informatik.rwthaachen.de/Publications/CEUR-WS/Vol-18

Gruber, T.R., 1993. A Translation Approach to Portable Ontology Specifications, Knowledge Acquisition, v.5, no. 2, Academic Press, pp. 199-220.

Horridge, M., Knublauch, H., Rector, A., Stevens, R., Wroe, C., 2004. A Practical Guide to Building OWL Ontologies Using the Protégé-OWL Plugin and CO-ODE Tools (Edition 1.0). The University of Manchester, 27 August 2004. Available online:

Iosifescu-Enescu, I., Hurni, L., 2007 Towards cartographic ontologies or "how computers learn cartography", In: Proceedings $23^{\text {rd }}$ International Cartographic Conference, 4-10 August 2007, Moscow, Russia.

Kemp, Z., 2004. A Knowledge-based Collaborative Environment for Geovisualization: Ontologies for Multiple Perspectives on Distributed Data Resources (2004). In: MacEachren, A.; Dykes, J.; Kraak, M.J. (eds.) Exploring Geovisualization. Elsevier, Oxford.

Kulik, L., Duckham, M., Egenhogfer, M.J., 2005. Ontology-driven Map Generalization. Journal of Visual Languages and Computing, v. 16 (2), pp. $245-267$

Lacy, L.W., 2005. OWL: representing information using the web ontology language. Trafford Publishing.

Lüscher, P., Burghardt, D., Weibel, R., 2007. Ontology-driven Enrichment of Spatial Databases. In: $10^{\text {th }}$ ICA Workshop on Generalisation and Multiple Representation, Moscow, Russia. http://ica.ign.fr/BDpubli/moscow2007/ Luescher-ICAWorkshop.pdf

Noy, N.F., McGuinness, D.L., 2001. Ontology Development 101: A Guide to Creating Your First Ontology. Stanford Knowledge Systems Laboratory Technical Report KSL-01-05 and Stanford Medical Informatics Technical Report SMI-2001-0880. http://protege.stanford.edu/publications/ ontology_development/ontology101.pdf

Peterson, G.N., 2009. GIS Cartography: a Guide to Effective Map Design. Taylor \& Francis.

Regnauld, N., 2007. Evolving from Automating Existing Map Production Systems to Producing Maps on Demand Automatically. In: $10^{\text {th }}$ ICA Workshop on Generalisation and Multiple Representation, Moscow, 2-3 August 2007). http://ica.ign.fr/BDpubli/moscow2007/RegnauldICAWorkshop.pdf

Robinson, A.H., Morrison, J.L., Muehrcke, P.C., Kimerling, A.J., Guptil, S.C., 1995. Elements of Cartography ( $6^{\text {th }}$ Ed.), Wiley NewYork.

Russell, S. J., Norvig, P., 2009. Artificial Intelligence: A Modern Approach ( $3^{\text {rd }}$ Ed.). Prentice Hall, Upper Saddle River, NJ.

Slocum, T.A., McMaster, R.B., Kessler, F.C., Howard, H.H., 2009. Thematic Cartography and Geovisualization ( ${ }^{\text {rd }}$ Ed.). Prentice Hall, Upper Saddle River, NJ.

Smith, M.K., Welty, C., McGuinness, D.L., 2004. OWL Web Ontology Language Guide. W3C Recommendation. http://www.w3.org/TR/owlguide/

Svátek, V., Labský, M., 2003. Objektové modely a znalostní ontologie podobnosti a rozdíly. In: Proceedings Objekty 2003, Ostrava. http:// nb.vse.cz/ svatek/obj03fi.pdf

Svátek, V., Zamazal, O., Presutti, V., 2009. Ontology Naming Pattern Sauce for (Human and Computer) Gourmets. In: Proceedings WOP 2009, Washington, pp. 171-178

Swartout, B., Patil, R., Knight, K., Russ, T., 1996. Toward distributed use of large-scale ontologies. In: Proceedings of the Tenth Knowledge Acquisition for Knowledge-Based Systems Workshop (KAW ' 96 November 9-14, Banff, Alberta, Canada). http://www.aaai.org/Papers/ Symposia/Spring/1997/SS-97-06/SS97-06-018.pdf.

Uschold, M., Grüninger, M., 1996. Ontologies: principles, methods and applications. The Knowledge Engineering Review, Cambridge University Press, Vol.11:2, pp. 93-136.

Wolf, E. B., 2009. Ontology-Driven Generalization of Cartographic Representations by Aggregation and Dimensional Collapse. In: The Semantic WEB - ISWC 2009. LNCS, Springer, Heidelberg, vol. 5823/ 2009, pp. 990-997. 